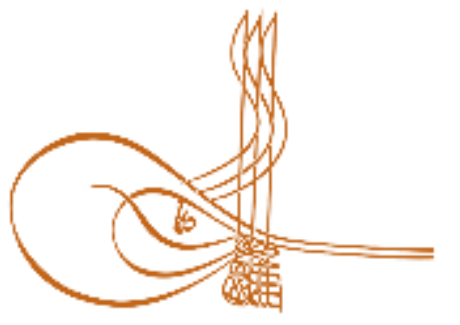

www.turkishstudies.net/turkishstudies
Turkish Studies

eISSN: $1308-2140$

Research Article / Araştırma Makalesi

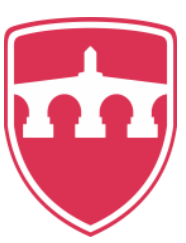

INTERNATIONAL

BALKAN

UNIVERSITY

Sponsored by IBU

\title{
Alt Ekstremite Y Dinamik Denge Testi Güvenirlik Çalışması
}

\author{
The Study on the Reliability of Lower Extremity Y Dynamic Balance Test
}

\author{
Cenab Türkeri ${ }^{*}$ Bilgihan Büyüktaş** - Barışcan Öztürk ${ }^{* * *}$
}

\begin{abstract}
This study was carried out to determine the measurement reliability of the lower extremity $\mathrm{Y}$ dynamic balance test, which is applied by keeping the hands still at the waist. In our study, the lower extremity Y dynamic balance test that we examined for reliability. This test provides data collection from the athlete using trunk rotation, limb mobility, ankle instability and flexibility of the lower limb. According to these data, it provides an estimate of the athlete's balance asymmetry and susceptibility to injuries. The study was conducted on a total of 75 volunteer athletes that live in Adana, from different sports branches, whose sports age was $9.06 \pm 3.86$ years, age $22.76 \pm 2.73$ years, height $174 \pm 0.08 \mathrm{~cm}$, weight $69.63 \pm 12.71 \mathrm{~kg}$. KolmogorovSmirnov test was applied for normality distribution of the data and it was found that the data showed normal distribution. The relationship between the lower extremity Y dynamic balance test scores performed at two weeks' intervals and at the same time of the day was found with the Pearson product-moment correlation coefficient. Intraclass Correlation Coefficient (ICC) test (95\% confidence intervals) was also used to determine the class reliability of the test. According to the findings of the study; The first and second measurements of the lower extremity $Y$ dynamic balance test were found to be high related (right composite $r=0.83$ ( $r 2=0.69$ ), left composite $\mathrm{r}=0.82(\mathrm{r} 2=0.67))$. Y dynamic balance test was found to have high Intraclass Correlation Coefficients (right $=0.91$ and left $=0.90 \mathrm{ICC}$ ). It was found that the measurement reliability of the lower extremity Y dynamic balance test, which was applied by keeping the hands fixed at the waist, was high. Dynamic balance performance can be evaluated with this test. In addition, it can be said that it can be used in both research and applications in order to determine the balance asymmetry of the athlete and predict the predisposition to sports injuries.
\end{abstract}

\footnotetext{
* Dr. Öğr. Üyesi, Çukurova Üniversitesi, Beden Eğitimi ve Spor Yüksekokulu, Antrenörlük Eğitimi Bölümü Asst. Prof. Dr., Cukurova University, School of Physical Education and Sports, Department of Coaching Education ORCID 0000-0003-4850-9810 cturkeri@cu.edu.tr

** Yüksek Lisans Öğr. Çukurova Üniverstesi, Sağlık Bilimleri Enstitüsü, Beden Eğitimi Spor Anabilim Dalı Graduate Student, Cukurova University, Institute of Medical Sclences, Department of Physical Education and Sports ORCID 0000-0002-6816-7275

bilgihanbykts10@gmail.com

**** Yüksek Lisans Öğr. Çukurova Üniverstesi, Sağlık Bilimleri Enstitüsü, Beden Eğitimi Spor Anabilim Dalı Graduate Student, Cukurova University, Institute of Medical Sclences, Department of Physical Education and Sports ORCID 0000-0001-7001-3032

bariscan.ozturk.bc@gmail.com

Cite as/ Atıf: Türkeri, C., Büyüktaş, B., Öztürk, B. (2020). Alt ekstremite y dinamik denge testi güvenirlik çalışması, Turkish Studies, 15(2), 1439-1451. https://dx.doi.org/10.29228/TurkishStudies.41683

Received/Geliş: 07 February/Şubat 2020

Accepted/Kabul: 25 April/Nisan 2020

Copyright (C) MDE, Turkey
} 
Structured Abstract: The Y dynamic balance test is a dynamic test that requires strength, flexibility, and proprioception. The purpose of the test is to try to reach as far as possible with the other leg while maintaining the fixed posture of the leg on one foot. In our study, the Lower Extremity Y dynamic balance test, whose reliability was examined; It provides data collection from the athlete using trunk rotation, limb mobility, ankle instability and flexibility of the lower extremity. According to the data, it provides an estimate of the athlete's balance asymmetry and susceptibility to injuries. Y dynamic balance test is very economical, easy to apply and can determine the balance asymmetry compared to other balance tests (Gribble, Hertel and Plisky, 2012). The fact that the test measuring device is easy to carry, simple application procedure, no breakage-distortion problem and can be used regardless of the laboratory or hall makes the test recommendable. During previous studies, measurements were taken by keeping the hands freely over the head. however, in our study, the lower extremity Y dynamic balance test was applied with the hands fixed at the waist and the measurement reliability was aimed. In this context, it is considered that the lower extremity Y dynamic balance test actively used will contribute to the researches in the field of movement and training sciences by determining the reliability of the measurements to be held by keeping the hands on the waist.

Our research was carried out on 75 volunteer athletes (female $=17$, male $=58$ ) who were all in the city of Adana, have a sports age of $9.06 \pm 3.86$ years and from different branches. Measurements were taken using the Y dynamic balance test device developed by Plisky.

Kolmogorov-Smirnov test was applied for normality distribution of the data and it was found that the data showed normal distribution. Relationships between Y dynamic balance test scores were determined with the Pearson Moments Product Correlation Coefficient. In addition, Intraclass Correlation Coefficient test (95\% confidence intervals) was used to determine the class reliability of the test.

When the demographic data of 75 athletes participating in this study were analyzed, it was observed that their average of age was $22.76 \pm 2.73$ years, height was $174 \pm 0.08 \mathrm{~cm}$, and weight was $69.63 \pm 12.71 \mathrm{~kg}$. Total values of Y dynamic balance measurements of athletes, right composite first measurement: $104.47 \pm$ 15.94 and second measurement: $108.68 \pm 19.04$, left composite first measurement: $104.50 \pm 15.64$ and second measurement: 109 It was found to be $86 \pm 18.15$. Considering the in-class reliability coefficient of the first and second measurements of the Lower Limb Y dynamic balance test, the highest values were found as right = 0.91 and left $=0.90 \mathrm{ICC}$.

The Y dynamic balance test is a test developed by Plisky et al (2006) instead of the star dynamic balance test (Star Excursion Balance Test). In our study, the lower extremity Y balance test, which we examine the measurement reliability, is applied by fixed the hands on the waist. The lower extremity Y dynamic balance test with this method obtains data on trunk rotation, limb mobility, ankle instability and flexibility of the lower extremity. Accordingly, it provides an estimate of the athlete's balance asymmetry and susceptibility to injuries.

In our country, recently, lower extremity $\mathrm{Y}$ dynamic balance test is used to determine the balance skills, mobility, ankle instability and / or balance asymmetries of athletes or sedentary individuals in sportive and clinical research (Haksever et al., 2017; Ateş, 2019; Koçak and Ünver, 2019; Kabul et al., 2018). However, despite the many studies, the reliability of the Lower Limb Y dynamic balance test with different arm position has not been studied. Accordingly, in order to eliminate the deficiencin the literature, it was aimed to determine the reliability of the Y dynamic balance test by using the test-retest method in athletes by fixed the hands on the waist.

In our study, it was found that the lower extremity Y dynamic balance test scores, which were applied by fixed the hands on the waist, had a high level of in-class reliability coefficient ( 0.86 to 0.91 ). When the average scores of the test are examined, it was seen that the scores obtained in both measurements were close to each other. This shows us that there was a significant consistency in the Y dynamic balance measurements made by fixed hands on the waist. Kinzey and Armstrong (1998) found in-class reliability coefficients at a high level (0.82-0.88) in the star dynamic balance test reliability study, which was a similar test. In a study conducted by Shaffer et al (2013), they found a high level of reliability in the application of the Y dynamic balance test with the hands freely method (0.80-0.91). Plisky et al. (2009) found the test to be very high reliable in a study, which examined the reliability of the Y dynamic balance test applied on the hands-free over the head. When the studies conducted are analyzed, the in-class reliability coefficient values obtained in our study are largely similar the other studies. 
When the results we obtained in our study are taken into consideration, it was seen that the measurement reliability of the $\mathrm{Y}$ dynamic balance test performed by fixed the hands on the waist was quite high in athletes. With the lower extremity Y dynamic balance test, data on trunk rotation, lower extremity mobility, ankle instability, and flexibility of the lower extremity can be obtained and dynamic balance performance can be evaluated. In addition, it can be said that the athlete can be used in both research and applications in order to estimate the susceptibility to injuries with balance asymmetry.

In order to support future studies, it may be recommended to examine the reliability of the lower and upper extremity Y dynamic balance tests together and determine the balance asymmetries. Also, it would be more accurate to compare the validity and reliability of the $\mathrm{Y}$ dynamic balance test with different electronic dynamic balance tests.

Keywords: Sport Science, Dynamic Balance, Lower Extremity, Mobility, Balance Asymmetry, Ankle Instability.

Öz: Bu çalışma Alt Ekstremite Y dinamik denge testinin eller belde sabit tutularak uygulanmasının ölçüm güvenirliğinin belirlenmesi amacıyla yapılmıştır. Çalışmamızda güvenirliğini incelediğimiz alt ekstremite $\mathrm{Y}$ dinamik denge testi; gövde rotasyonu, ekstremite hareketliliği, ayak bileği instabilitesini ve alt ekstremitenin esnekliğini kullanarak sporcudan veri toplanmasını sağlamakta ve buna göre sporcunun denge asimetrisi ve sakatlıklara yatkınlığı hakkında tahmini bilgi vermektedir. Araştırma tamamı Adana ilinde spor yapan, $9,06 \pm 3,86$ yıllık spor geçmişine sahip, yaşları 22,76 $\pm 2,73$ yıl, boyları $174 \pm 0,08 \mathrm{~cm}$, ağırlıkları $69,63 \pm 12,71 \mathrm{~kg}$ olan farklı branşlardan toplam 75 gönüllü sporcu (kadın=17, erkek=58) üzerinde yürütülmüştür. Verilerin normallik dağılımı için Kolmogorov-Smirnov testi uygulanmış ve verilerin normal dağılım gösterdiği bulunmuştur. İki hafta arayla günün aynı saatleri arasında yapılan Y dinamik denge test skorları arasındaki ilişki Pearson Momentler Çarpımı Korelasyon Katsayısı ile bulunmuş ve ayrıca testin sınıf içi güvenirliğinin belirlenmesinde Intraclass Correlation Coefficient testi (95\% confidence intervals) uygulanmıştır. Çalışmadan elde edilen bulgular doğrultusunda alt ekstremite $Y$ dinamik denge testi ilk ve ikinci ölçümleri arasında yüksek ilişki bulunmuştur ( sağ composite $r=0,83\left(r^{2}=0,69\right)$, sol composite $\left.r=0,82\left(r^{2}=0,67\right)\right)$. Y dinamik denge testi sınıf içi güvenirlik katsayısı yüksek bulunmuştur (sağ=0,91 ve sol=0,90). Alt ekstremite $\mathrm{Y}$ dinamik denge testinin eller belde sabit tutularak uygulamasının ölçüm güvenirliğinin yüksek olduğu ve bu testle dinamik denge performansının değerlendirilebileceği, bunun yanı sıra sporcunun denge asimetrisi ile sakatlıklara yatkınlığının tahmin edilebilmesi amacıyla hem araştırmalarda hem de uygulamalarda kullanılabileceği söylenebilir.

Anahtar Kelimeler: Spor Bilimleri, Dinamik Denge, Alt Ekstremite, Hareketlilik, Denge Asimetrisi, Ayak Bileği İnstabilitesi

\section{Giriş}

Sportif performansın temelini oluşturan, kondisyonel becerilerin merkezinde bulunan denge becerisinin birçok sporsal becerinin başarılı sergilenmesinde, yön değiştirmede, durmada, başlamada, tutma konusunda, nesneyi hareket ettirmede, vücudun belli pozisyonun korunmasinda aktif rol oynamaktadır (Erdoğan ve ark., 2017). İnsanın denge sağlamadaki yeteneği, diğer motor sistemlerin gelişmesinde belirleyici bir faktör olarak tanımlanabilir (Erkmen ve ark., 2007). Sporcuların sportif performanslarını meydana getirme de önemli bir etken olan dinamik denge, aynı zamanda da sporcuların alt ekstremite yaralanmalarında performansı belirleyici ve önleyici bir etkiye sahip motorsal bir beceridir (Butler ve ark.,2013; Gribble, Hertel ve Plisky, 2012; Plisky ve ark., 2009). Çok sayıda spor kurumu, sporcular için uygun tarama testlerinin eksik olduğunu belirtmektedir (Batt, Jaqueve Stone, 2004; Fuller, Ojelade ve Taylor, 2007). Ayrica, bu tür testlerin hedeflenen tarama popülasyonu için güvenilir, hassas, spesifik, ekonomik ve kabul edilebilir olması gerektiğini belirtmektedir (Ljungqvist ve ark., 2009). Dinamik denge testi, artan sakatlanma risklerini belirlemek amacıyla kas iskelet sakatlanma sisteminin bir bileşeni olarak popülerlik kazanmaktadır (Dennis ve ark., 2008; Kiesel, Plisky ve Voight, 2007; Plisky ve ark., 2009; Plisky ve ark., 2006). Bilinen sakatlanma risk faktörleri arasında antropometrik özellikler, mevki (pozisyon), yetenek 
düzeyi, antrenman süresi, esneklik, asimetri, geçmiş sakatlanma durumu, saha yüzeyi ve tercih edilen ayakkabı türü bulunmaktadır (Dick ve ark., 2007; Faude ve ark., 2006; Giza ve ark., 2005; Heidt ve ark., 2000). Bu risk faktörleri ayrı ayrı tanımlanmış olsa da, sakatlanma riskini bir bütün olarak etkilemektedir. Son zamanlarda, araştırmacılar sakatlanmayı tahmin etmek için kapsamlı hareket kalıplarını içeren testleri kullanmıştır (Kiesel, Plisky ve Voight, 2007; Plisky ve ark., 2006).

$\mathrm{Bu}$ hareket kalıplarını içeren bir test ise $\mathrm{Y}$ dinamik denge testidir. $\mathrm{Y}$ dinamik denge testi güç, esneklik ve propriosepsiyon gerektiren dinamik bir testtir. Testin amacı, bacağın tek ayak üzerinde sabit duruşunu korurken diğer bacak ile mümkün olduğunca ileri noktaya ulaşmaya çalışmaktır (Kienzey ve Armstrong, 1998). Y dinamik denge testi farklı branşlardaki sporcuların fiziksel performansını ölçmek, denge becerilerini karşılaştırmak ve sporcuların kronik ayak bileği instabilitesini belirlemek için kullanılmaktadır (Hale, Hertel, Olmsted-Kramer, 2007; Olmsted ve ark., 2002).

Y dinamik denge testi alt ekstremite ve üst ekstremite için farklı şekillerde olmak üzere kullanılabilmektedir. Çalışmamızda güvenirliğini incelediğimiz alt ekstremite Y dinamik denge testi; gövde rotasyonu, ekstremite hareketliliği, ayak bileği instabilitesini ve alt ekstremitenin esnekliğini kullanarak sporcudan veri toplanmasını sağlamakta ve buna göre sporcunun denge asimetrisi ve sakatlıklara yatkınlığ 1 hakkında tahmini bilgi vermektedir. Literatürde belirtildiği gibi sporda dinamik denge, başarılı performansın göstergelerinden biri olarak kabul edilir. Sporcunun dinamik denge becerisinin iyi olması sporcunun müsabaka ya da antrenman anında branşının gerektirdiği teknik beceri uygulama durumlarında veya ikili mücadele anlarında sporcunun bulunduğu pozisyonda sporcunun, dengede kalmasını, postural salınımını düzenlemesine dolayısıyla oluşabilecek sakatlıkları önleyebilmesi ve başarılı performans sergileyebilmesi için gerekli ögelerden biridir. Bundan dolayı, sporcunun dinamik dengesinin değerlendirilmesi ve eğer dinamik denge becerisinde bir eksiği varsa düzeltici egzersizlerin verilmesi gerekmektedir.

Y dinamik denge testi, diğer denge testlerine kıyasla çok ekonomik, uygulaması kolay ve denge asimetrisini belirleyebilen bir testtir (Gribble, Hertel ve Plisky, 2012). Test ölçüm düzeneğinin kolay taşınabilir olması, basit uygulama izleği, kırılma-bozulma probleminin olmaması ve açıkkapalı hemen her ortamda kullanılabilir olması testi tavsiye edilebilir kılmaktadır. Belirtilen avantajlarından dolayı bu test seçilmiştir. Daha önceki çalışmalar sırasında eller serbest olarak baş üstünde tutularak ölçümler alınmıştır ancak çalışmamızda alt ekstremite $\mathrm{Y}$ dinamik denge testi eller belde sabit tutularak ölçümler alınarak sporcularda ölçüm güvenirliğinin belirlenmesi amaçlanmıştır. Bu bağlamda aktif olarak kullanılan alt ekstremite $Y$ dinamik denge testinin eller belde sabit tutularak sporcularda ölçüm güvenirliğinin belirlenmesiyle hareket ve antrenman bilimleri alanında yapılacak olan araştırmalara katkı sağlayacağı düşünülmektedir.

\section{Gereç ve Yöntem}

\section{Kattlimcilar}

Çukurova Üniversitesi, BESYO, Performans Ölçüm Laboratuvarında yapılan çalışmaya, herhangi bir yaralanması olmayan, branşında $9,06 \pm 3,86$ yıllık spor geçmişine sahip, yaş ortalamaları $22,76 \pm 2,73 \mathrm{y} 1$, boy ortalamaları $1,74 \pm 0,08 \mathrm{~cm}$, ağırlı ortalamaları $69,63 \pm 12,71 \mathrm{~kg}$ farklı spor branşlardan toplam 75 sporcu ( $k$ adın=17 ve erkek=58) gönüllü olanlar arasından rastgele seçilerek alınmıştır. Sporculara çalışma protokolü hakkında bilgi verilmiş, kabul edenlere çalışmanın amaç ve yöntemlerini belirten onam formu imzalatılmıştır. Çukurova Üniversitesi girişimsel olmayan klinik araştırmalar etik kurulundan 10.01.2020/ tpl: 95/ karar: 56 tarih ve sayı ile onay alınmıştır.

\section{Verilerin Toplanmast}

Katılımcıların demografik özellikleri için araştırmacılar tarafından hazırlanan anket formu kullanılmıştır. Formda kişinin; cinsiyet, yaş, boy, ağırlık, bacak boyu, spor yaşı ve body mass index (BMI) verileri kaydedilmiştir. Sporcuların hastalık, yaralanma, ameliyat varlığı, kullandığı ilaçlar, 
alerji ve beslenme durumları sorgulanmış, herhangi bir sorunu olmayanlar antrenman yapmadıkları bir günün ardından önce ilk ölçüme alınmış ve ardından iki hafta sonra yine antrenman yapmadıkları günün ardından aynı gün ve saatlerde ikinci ölçümleri aynı araştırmacı tarafından alınmıştır.

\section{Y Dinamik Denge Test Materyali}

Alt Ekstremite Y Dinamik Denge Test materyali, merkez bir platforma (denge noktası) bağlı anterior, posteromedial ve posterolateral uzanma yönlerine sahip üç adet PVC borudan ve bu borulara yerleştirilmiş üç adet PVC bloktan oluşmaktadır. Platformun arka yönlerindeki (posteromedial ve posterolateral) borular kendi arasında $45^{\circ}$, anterior yöndeki borudan ise $135^{\circ}$ olacak şekilde konumlandırılmıştır. Sporcuların erişebildikleri mesafeyi belirlemek için platforma bağlı olan anterior, posteromedial ve posterolateral yönlerdeki boruların üzerinde $1 \mathrm{~mm}$ aralıklarda mesafe ölçer bulunmaktadır.

\section{Alt Ekstremite Y Dinamik Denge Testi Uygulama Protokolü}

Alt Ekstremite Y dinamik denge Testi, iki hafta aralıkla aynı gün ve aynı saatlerde Çukurova Üniversitesi BESYO Performans Ölçüm Laboratuvarında uygulanmıştır. Test, deneklerin (dominant ve non-dominant) her iki ayakla ayrı uygulanmıştır. Denekler hareketlerini kısıtlamayacak spor giysileriyle, ayakları çıplak olarak, Y dinamik denge test platformunun üzerinde eller bel bölgesinde sabit tutularak ve ayağı merkez noktada sabit bir şekilde dengede durmuştur. Daha sonra bir ayağı ile sabit duruşunu koruyarak, diğer ayağıyla anterior $\left(0^{\circ}\right)$, posteromedial $\left(45^{\circ}\right)$ ve posterolateral $\left(45^{\circ}\right)$ yönlere doğru ayak parmak ucu ile blokları (her seferinde sabit duruş noktasına ayağı yere değdirmeden geri getirerek) itmiştir (şekil 2). Test her üç yönde (anterior, posteromedial, posterolateral) $3 \mathrm{kez}$ tekrar edildikten sonra normalleştirme formülü kullanılmıştır (şekil 2).

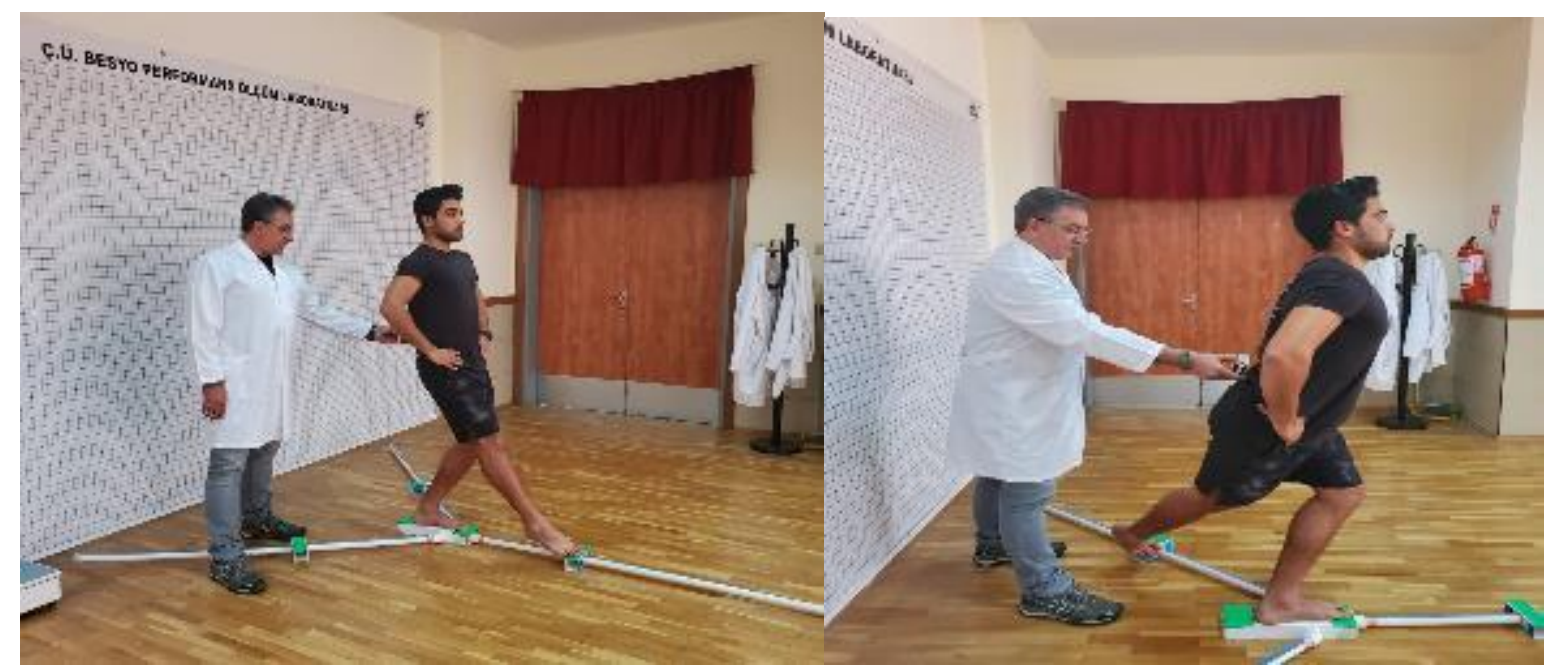

Şekil 1. Alt Ekstramite Y Dinamik Ölçüm Düzeneği

Anterior+Posteromedial+Posterolateral

$3 \times$ Limb Length

Şekil 2. Ölçümlerin Normalleştirme Formülü 


\section{İstatistiksel Analiz}

İstatistiksel analizler, SPSS 22.0 programı kullanılarak yapılmıştır. Deneklerin demografik özellikleri tanımlayıcı istatistikler ile analiz edilmiştir. Sonuçları, aritmetik ortalama \pm standart sapma $(\overline{\mathrm{X}} \pm$ ss $)$ olarak verilmiştir. Verilerin normallik dağılımı için Kolmogorov-Smirnov testi uygulanmış ve normal dağılım gösterdiği bulunmuştur. Y dinamik denge test skorları arasındaki ilişki Pearson Momentler Çarpımı Korelasyon Katsayısı ile bulunmuş, ayrıca testin sınıf içi güvenirliğinin belirlenmesinde Intraclass Correlation Coefficient (ICC) testi (95\% confidence intervals) uygulanmıştır.

\section{Bulgular}

Tablo 1'de çalışmaya katılan kadın $17(\% 22,7)$ ve erkek $58(\% 77,3)$ toplam 75 sporcunun demografik verileri incelendiğinde, yaş ortalamalarının $22,76 \pm 2,73$ yıl, boylarının $174 \pm 0,08 \mathrm{~cm}$ olduğu, vücut ağırlıklarının $69,63 \pm 12,71 \mathrm{~kg}$ ve spor yaşlarının $9,06 \pm 3,86$ yıl olduğu görülmüsştür.

Tablo 1: Cinsiyete Göre Sporcuların Demografik Özellikleri

\begin{tabular}{lccc}
\hline \multicolumn{1}{c}{$\mathbf{n = 7 5}$} & $\begin{array}{c}\text { Kadın } \\
\overline{\mathbf{x}} \pm \mathbf{s}\end{array}$ & $\begin{array}{c}\text { Erkek } \\
\overline{\mathbf{x}} \pm \mathbf{s s}\end{array}$ & $\begin{array}{c}\text { Toplam } \\
\overline{\mathbf{x}} \pm \mathbf{s s}\end{array}$ \\
\hline Yaş (yıl) & $22,76 \pm 3,91$ & $22,76 \pm 2,32$ & $22,76 \pm 2,73$ \\
Boy (cm) & $165 \pm 0,06$ & $177 \pm 0,06$ & $174 \pm 0,08$ \\
Vücut Ağırlı̆̆ı (kg) & $56,00 \pm 10,16$ & $73,62 \pm 10,45$ & $69,63 \pm 12,71$ \\
Spor Yaşı (yıl) & $8,27 \pm 3,71$ & $9,31 \pm 3,90$ & $9,06 \pm 3,86$ \\
Bacak Boyu (cm) & $88,77 \pm 6,04$ & $92,31 \pm 6,91$ & $91,51 \pm 6,85$ \\
BMI & $20,43 \pm 2,91$ & $23,46 \pm 2,51$ & $22,78 \pm 2,89$ \\
\hline
\end{tabular}

Tablo 2'de sporcuların branşlara göre dağılımı verilmiştir. Buna göre takım sporları n=35 $(\% 46,7)$ ve bireysel sporlar $n=40(\% 53,3)$ olduğu görülmektedir.

Tablo 2: Sporcuların Branşlara Göre Dağılımı

\begin{tabular}{llll}
\hline $\mathbf{n = 7 5}$ & BRANŞ & $\mathbf{n}$ & $\mathbf{\%}$ \\
\hline & Futbol & 18 & 24,0 \\
Takım Spor Branşları & Basketbol & 6 & 8,0 \\
$\mathbf{n = 3 5}$ (\%46,7) & Voleybol & 3 & 4,0 \\
& Hentbol & 8 & 10,7 \\
\hline & Taekwondo & 12 & 16,0 \\
\multirow{3}{*}{$\begin{array}{l}\text { Bireysel Spor Branşları } \\
\text { n=40 (\%53,3) }\end{array}$} & Atletizm & 6 & 8,0 \\
& Cimnastik & 10 & 13,3 \\
& Karate & 7 & 9,3 \\
& Tenis & 5 & 6,7 \\
\hline & TOPLAM & $\mathbf{7 5}$ & $\mathbf{1 0 0 , 0}$ \\
\hline
\end{tabular}

Tablo 3'te çalışmaya katılan sporcuların kadın, erkek ve toplam Y dinamik denge ölçümleri

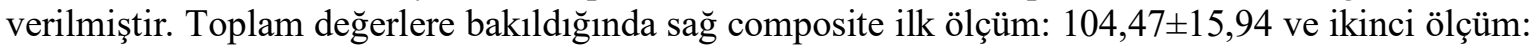

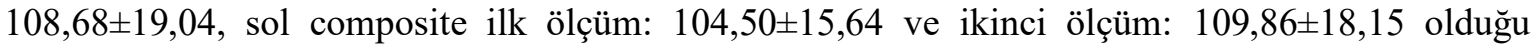
bulunmuştur. 
Tablo 3: Kadın ve Erkek Sporcuların 1. Ölçüm ve 2. Ölçüm Y Dinamik Denge Skorları

\begin{tabular}{|c|c|c|c|c|c|c|c|}
\hline & \multirow[t]{2}{*}{$n=75$} & \multicolumn{2}{|c|}{ Kadın } & \multicolumn{2}{|c|}{ Erkek } & \multicolumn{2}{|c|}{ Toplam } \\
\hline & & 1.Ölçüm & 2. Ölçüm & 1.Ölçüm & 2. Ölçüm & 1.Ölçüm & 2. Ölçüm \\
\hline & & $\overline{\mathbf{x}} \pm \mathbf{S S}$ & $\overline{\mathbf{X}} \pm \mathbf{s S}$ & $\overline{\mathbf{X}} \pm \mathbf{S S}$ & $\overline{\mathbf{X}} \pm \mathbf{S S}$ & $\overline{\mathbf{X}} \pm \mathbf{S S}$ & $\overline{\mathbf{X}} \pm \mathbf{s S}$ \\
\hline \multirow{4}{*}{$\sum_{\infty}^{U}$} & Anterior & $65,35 \pm 10,64$ & $68,79 \pm 12,02$ & $74,78 \pm 11,84$ & $79,92 \pm 13,45$ & $72,65 \pm 12,18$ & $77,40 \pm 13,88$ \\
\hline & Post-medial & $95,76 \pm 14,74$ & $98,06 \pm 15,94$ & $109,40 \pm 16,56$ & $113,67 \pm 18,84$ & $106,31 \pm 17,07$ & $110,15 \pm 19,28$ \\
\hline & Post-Lateral & $94,62 \pm 14,42$ & $94,71 \pm 16,07$ & $110,33 \pm 17,37$ & $113,61 \pm 18,35$ & $106,77 \pm 17,92$ & $109,32 \pm 19,46$ \\
\hline & Composite & $96,44 \pm 13,96$ & $98,57 \pm 15,79$ & $106,82 \pm 15,81$ & $111,64 \pm 19,01$ & $104,47 \pm 15,94$ & $108,68 \pm 19,04$ \\
\hline \multirow{4}{*}{$\bar{\partial}$} & Anterior & $65,50 \pm 11,28$ & $70,56 \pm 13,60$ & $75,36 \pm 11,94$ & $80,36 \pm 14,72$ & $73,13 \pm 12,43$ & $78,14 \pm 14,97$ \\
\hline & Post-medial & $95,38 \pm 15,63$ & $96,15 \pm 16,22$ & $109,92 \pm 16,13$ & $115,03 \pm 17,92$ & $107,63 \pm 17,05$ & $110,75 \pm 19,17$ \\
\hline & Post-Lateral & $95,50 \pm 15,86$ & $97,44 \pm 17,40$ & $109,28 \pm 16,94$ & $115,61 \pm 17,13$ & $107,16 \pm 17,58$ & $111,49 \pm 18,71$ \\
\hline & Composite & $96,58 \pm 14,25$ & $99,41 \pm 15,18$ & $106,82 \pm 15,37$ & $112,93 \pm 17,91$ & $104,50 \pm 15,64$ & $109,86 \pm 18,15$ \\
\hline
\end{tabular}

Tablo 4'te çalışmaya katılan sporcuların ilk ve ikinci ölçüm Alt Ekstremite Y dinamik denge sınıf içi güvenirlik katsayıları verilmiştir. Alt Ekstremite $\mathrm{Y}$ dinamik denge testi ilk ve ikinci ölçümleri sınıf içi güvenirlik katsayısı en yüksek ( $\mathrm{sağ}=0,91$ ve sol=0,90 ICC) olarak bulunmuştur.

Tablo 4: Sporcuların İlk ve İkinci Ölçüm Y Dinamik Denge Test Skorlarının Güvenirlik Sonuçları

\begin{tabular}{lllll}
\hline $\mathrm{n}=75$ & & ICC & $\mathbf{9 5 \%}$ CI & SEM \\
\hline \multirow{4}{*}{ SA $\breve{G}$} & Anterior & 0,91 & $0,87-0,95$ & $1,41-1,60$ \\
& Post-medial & 0,89 & $0,83-0,93$ & $1,97-2,23$ \\
& Post-Lateral & 0,86 & $0,78-0,91$ & $2,07-2,25$ \\
& Composite & 0,89 & $0,83-0,93$ & $1,84-2,20$ \\
\hline \multirow{3}{*}{ SOL } & Anterior & 0,90 & $0,84-0,94$ & $1,44-1,73$ \\
& Post-medial & 0,89 & $0,83-0,93$ & $1,97-2,21$ \\
& Post-Lateral & 0,89 & $0,83-0,93$ & $2,03-2,16$ \\
& Composite & 0,90 & $0,83-0,93$ & $1,81-2,10$ \\
\hline
\end{tabular}

ICC: Intra Class Coefficient, CI: Confidence Interval, SEM: Standart Error Means

Tablo 5 'te çalışmaya katılan sporcuların alt ekstremite Y dinamik denge güvenirlik sonuçları verilmiştir. Tabloya göre, sağdan alınan ölçümlerde; anterior1-anterior2 $r=0,85 \quad\left(r^{2}=0,72\right)$, posteromedial1-posteromedial2 $r=0,82\left(r^{2}=0,67\right)$, posterolateral1-posterolateral2 $r=0,76\left(r^{2}=0,58\right)$ composite1-composite $2 \mathrm{r}=0,83\left(\mathrm{r}^{2}=0,69\right)$ olarak bulunmuştur. Soldan alınan ölçümlerde; anterior 1 anterior2 $\mathrm{r}=0,83 \quad\left(\mathrm{r}^{2}=0,69\right)$, posteromedial1-posteromedial2 $\mathrm{r}=0,81 \quad\left(\mathrm{r}^{2}=0,66\right)$ posterolateral1posterolateral $2 \mathrm{r}=0,80\left(\mathrm{r}^{2}=0,64\right)$ composite 1 -composite $2 \mathrm{r}=0,82\left(\mathrm{r}^{2}=0,67\right)$ olarak bulunmuştur. 
Cenab Türkeri - Bilgihan Büyüktaş - Barışcan Öztürk

Tablo 5: Sporcuların Alt Ekstremite Y dinamik Denge 1. Ölçüm ve 2. Ölçüm Korelasyon Tablosu

\begin{tabular}{|c|c|c|c|c|c|c|}
\hline & $n=75$ & & $\begin{array}{l}\text { ANTERİOR } \\
2\end{array}$ & $\begin{array}{c}\text { POSTEROMEDİAL } \\
2\end{array}$ & $\begin{array}{l}\text { POSTEROLATERAL } \\
2\end{array}$ & COMPOSITE \\
\hline \multirow{8}{*}{ 岁 } & ANTERIOOR & $\mathrm{r}$ & $0,851^{* *}$ & $0,531^{* *}$ & $0,490^{* *}$ & $0,589^{* *}$ \\
\hline & 1 & $\mathrm{p}$ & 0,000 & 0,000 & 0,000 & 0,000 \\
\hline & POSTEROMEDİAL & $\mathrm{r}$ & $0,478^{* *}$ & $0,815^{* *}$ & $0,684^{* *}$ & $0,613^{* *}$ \\
\hline & 1 & $\mathrm{p}$ & 0,000 & 0,000 & 0,000 & 0,000 \\
\hline & POSTEROLATERAL & $\mathrm{r}$ & $0,396^{* *}$ & $0,723^{* *}$ & $0,759^{* *}$ & $0,522^{* *}$ \\
\hline & 1 & $\mathrm{p}$ & 0,000 & 0,000 & 0,000 & 0,000 \\
\hline & COMPOSITE & $\mathrm{r}$ & $0,614^{* *}$ & $0,723^{* *}$ & $0,624^{* *}$ & $\mathbf{0 , 8 2 8}{ }^{* * *}$ \\
\hline & 1 & $\mathrm{p}$ & 0,000 & 0,000 & 0,000 & 0,000 \\
\hline \multirow{8}{*}{$\overrightarrow{0}$} & ANTERIOR & $\mathrm{r}$ & $0,832^{* *}$ & $0,453^{* * *}$ & $0,402^{* *}$ & $0,563^{* *}$ \\
\hline & 1 & $\mathrm{p}$ & 0,000 & 0,000 & 0,000 & 0,000 \\
\hline & POSTEROMEDİAL & $\mathrm{r}$ & $0,474^{* *}$ & $0,809^{* *}$ & $0,759^{* *}$ & $0,623^{* *}$ \\
\hline & 1 & $\mathrm{p}$ & 0,000 & 0,000 & 0,000 & 0,000 \\
\hline & \multirow{2}{*}{$\begin{array}{c}\text { POSTEROLATERAL } \\
1\end{array}$} & $\mathrm{r}$ & $0,418^{* *}$ & $0,671^{* *}$ & $0,804^{* *}$ & $0,547^{* *}$ \\
\hline & & $\mathrm{p}$ & 0,000 & 0,000 & 0,000 & 0,000 \\
\hline & COMPOSITE & $\mathrm{r}$ & $0,644^{* *}$ & $0,644^{* *}$ & $0,587^{* *}$ & $\mathbf{0 , 8 2 0}{ }^{* *}$ \\
\hline & 1 & $\mathrm{p}$ & 0,000 & 0,000 & 0,000 & 0,000 \\
\hline
\end{tabular}

$* * \mathbf{p}<0,01$

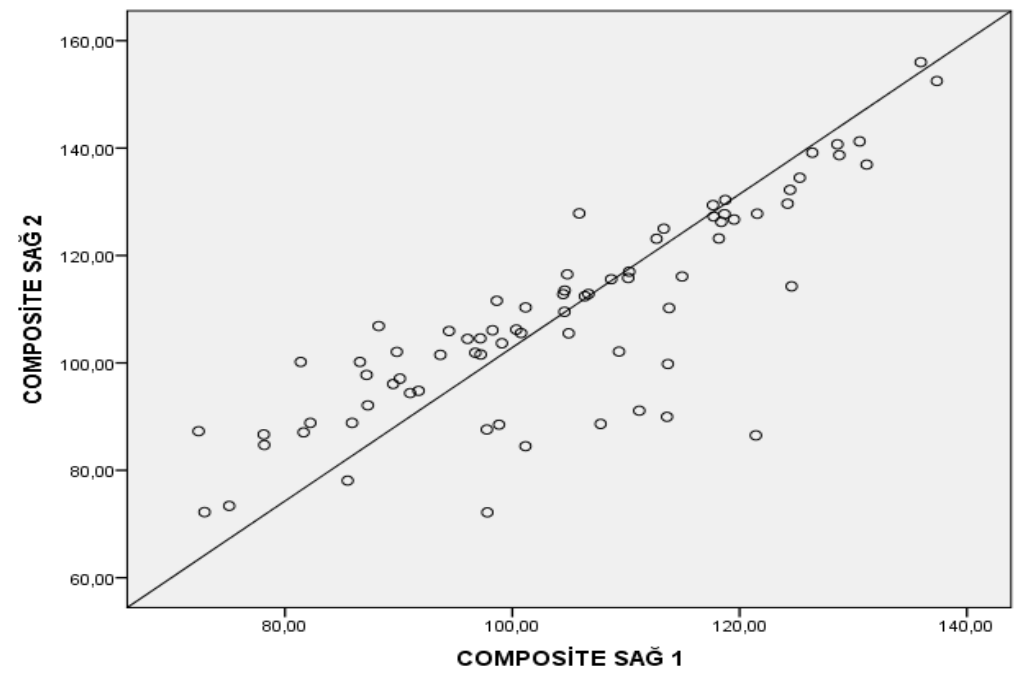

Turkish Studies, 15(2) 


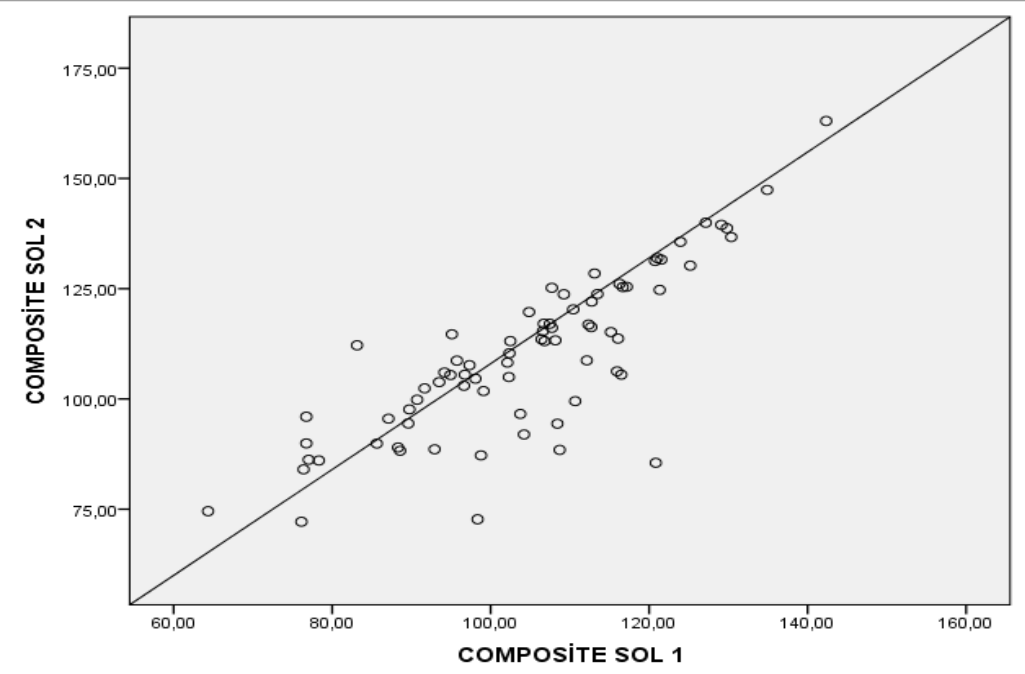

Şekil 3. Sağ Composite 1 -2 ve Sol Composite1-2 İlişki Grafikleri

\section{Tartışma}

Y dinamik denge testi Plisky et al (2006) tarafından yıldız dinamik denge testi (Star Excursion Balance Test) yerine geliştirilmiş bir testtir. Y dinamik denge testi, Yıldız dinamik denge testine göre kullanımı daha kolay ve daha doğru sonuç verdiği düşünülmektedir. Y dinamik denge testi alt ekstremite ve üst ekstremite için farklı şekillerde olmak üzere kullanılabilmektedir. Çalışmamızda güvenirliğini incelediğimiz Alt Ekstremite Y dinamik denge testi (eller belde sabit tutularak); gövde rotasyonu, ekstremite hareketliliği, ayak bileği instabilitesini ve alt ekstremitenin esnekliğini kullanarak sporcudan veri toplanmasını sağlamakta ve buna göre sporcunun denge asimetrisi ve sakatlıklara yatkınlığı hakkında tahmini bilgi vermektedir.

Yapılacak olan çalışmalarda kullanılan ölçüm aleti ya da ölçüm yöntemlerinin kararlı özellik taşıması, hatadan arındırılmış olması ve aynı amaçla yapılacak ikinci bir ölçümde aynı sonuçların elde edilebileceğine güven duyulması yapılacak olan çalışmaların güvenirliğini arttırmaktadır (Ercan ve Kan, 2004). Ülkemizde son zamanlarda, sportif ve klinik araştırmalarda sporcuların ya da sedanter bireylerin denge becerilerini, hareketliliklerini, ayak bileği instabilitesini ve/veya denge asimetrilerini belirlemek amaciyla alt ekstremite Y dinamik denge testi kullanılmaktadır (Haksever ve ark., 2017; Ateş, 2019; Koçak ve Ünver, 2019; Kabul ve ark., 2018). Ancak yapılan birçok çalışmaya rağmen, Alt Ekstremite Y dinamik denge testinin farklı el pozisyonuyla güvenirliği üzerine çalışma yapılmamıştır. Bu doğrultuda literatürdeki eksikliği gidermek amacıyla Y dinamik denge testinin eller belde sabit tutularak sporcularda test-tekrar-test yöntemi kullanılarak güvenirliğinin belirlenmesi amaçlanmıştır.

Çalışmamızda eller belde sabit tutularak yapılan Y dinamik denge testinin 2 hafta ara ile uygulanması sonucu, Y dinamik denge testi skorlarının sınıf içi güvenirlik katsayılarının yüksek düzeyde ( 0.86 ile 0.91$)$ olduğu bulunmuştur. Testin, skor ortalamaları incelendiğinde her iki ölçümde elde edilen skorların birbirine yakın olduğu görülmektedir. Bu durum bize eller belde sabit tutularak yapılan Y dinamik denge ölçümlerinde önemli oranda tutarlılık olduğunu göstermektedir. Kinzey ve Armstrong (1998) benzer bir test olan Yıldız Dinamik Denge Testi güvenirlik çalışmasında sınıf içi güvenirlik katsayılarını yüksek düzeyde $(0,82-0,88)$ bulmuştur. Yapılan bir başka Yıldız denge testi güvenirlik çalışmasında Hertel, Miller ve Denegar (2000) ölçüm skorlarının sınıf içi güvenirlik katsayılarını $(0,78-0,96)$ bulmuştur. Shaffer ve arkadaşları (2013) eller serbest baş üstünde yapılan Y dinamik denge testinin güvenirliğini inceledikleri bir çalışmada dinamik denge skorlarını yüksek düzeyde $(0,80-0,91)$ bulmuştur. Plisky ve ark. (2009) eller serbest baş üstünde uygulanan Y dinamik denge test aracının güvenirliğini incelediği çalışmada testi çok yüksek düzeyde $(0,97-1,00)$ güvenilir 
bulmuştur. Yapılan çalışmalar incelendiğinde, elde edilen sınıf içi güvenirlik katsayı değerleri ile çalışmamızda elde edilen sınıf içi güvenirlik katsayı değerleri büyük oranda benzerlik göstermektedir.

\section{Sonuç}

Çalışmamızda elde ettiğimiz sonuçlar dikkate alındığında, eller belde sabit tutularak yapılan $\mathrm{Y}$ dinamik denge testinin sporcularda güvenirliğinin oldukça yüksek olduğu görülmektedir. $\mathrm{Bu}$ testle, gövde rotasyonu, alt ekstremite hareketliliği, ayak bileği instabilitesi ve alt ekstremitenin esnekliği ile ilgili verileri alınarak dinamik denge performansının değerlendirilmesi yapılabilir. Bunun yanı sıra sporcunun denge asimetrisi ile sakatlıklara yatkınlığının tahmin edilebilmesi amacıyla hem araştırmalarda hem de uygulamalarda kullanılabileceği söylenebilir.

\section{Öneriler}

Daha sonra yapılacak olan çalışmalara fikir vermesi amacıyla alt ve üst ekstremite Y dinamik denge testinin güvenirliğinin birlikte incelenip, denge asimetrilerinin belirlenmesi tavsiye edilebilir. Ayrıca, ilerleyen süreçlerde geçerliliği ve güvenirliği yüksek olan elektronik dinamik denge testleri ile Y dinamik denge testinin geçerliği ve güvenirliğinin karşılaştırılması daha doğru olacaktır.

\section{Kaynakça}

Bahar, A. (2019). Y denge test performansı ile hamstring esnekliği arasındaki ilişki. Gaziantep Üniversitesi Spor Bilimleri Dergisi, 4(1), 93-103. https://doi.org/10.31680/gaunjss.514993

Batt, M. E., Jaques, R., Stone, M. (2004). Preparticipation examination (screening): practical issues as determined by sport: a United Kingdom perspective. Clinical Journal of Sport Medicine, 14(3), 178-182. ISSN: 1050-642X. https://doi.org/10.1097/00042752200405000-00011

Butler, R. J., Lehr, M. E., Fink, M. L., Kiesel, K. B., Plisky, P. J. (2013). Dynamic balance performance and noncontact lower extremity injury in college football players: an initial study. Sports health, 5(5), 417-422. https://doi.org/10.1177/1941738113498703

Dennis, R. J., Finch, C. F., Elliott, B. C., Farhart, P. J. (2008). The reliability of musculoskeletal screening tests used in cricket. Physical Therapy in Sport,9(1), 25-33. https://doi.org/10.1016/j.ptsp.2007.09.004

Dick, R., Putukian, M., Agel, J., Evans, T. A., Marshall, S. W. (2007). Descriptive epidemiology of collegiate women's soccer injuries: National Collegiate Athletic Association Injury Surveillance System, 1988-1989 through 2002-2003. Journal of athletic training, 42(2), 278. PMID: 17710177. https://doi.org/10.1016/s0276-1092(08)79204-6

Ercan, İ., Kan, İ. (2004). Ölçeklerde güvenirlik ve geçerlik. Uludă̆ Üniversitesi Tıp Fakültesi Dergisi, 30(3), 211-216. ID: JA68ZN85VB.

Erdoğan, C. S., Fatmanur, E. R., İpekoğlu, G., Çolakoğlu, T., Zorba, E., Çolakoğlu, F. F. (2017). Farklı denge egzersizlerinin voleybolcularda statik ve dinamik denge performansı üzerine etkileri. Spor ve Performans Araştırmalart Dergisi, 8(1), 11-18. https://doi.org/10.17155/spd.20761

Erkmen, N., Suveren, S., Göktepe, A. S., Yazıcıoğlu, K. (2007). Farklı branşlardaki sporcuların denge performanslarının karşılaştırılması. Spormetre Beden Eğitimi ve Spor Bilimleri Dergisi, 5(3), 115-122. https://doi.org/10.1501/sporm_0000000080 
Faude, O., Junge, A., Kindermann, W., Dvorak, J. (2006). Risk factors for injuries in elite female soccer players. British journal of sports medicine, 40(9), 785-790. https://doi.org/10.1136/bjsm.2006.027540

Fuller, C. W., Ojelade, E. O., Taylor, A. (2007). Preparticipation medical evaluation in professional sport in the UK: theory or practice?. British journal of sports medicine, 41(12), 890-896. https://doi.org/10.1136/bjsm.2007.038935

Giza, E., Mithöfer, K., Farrell, L., Zarins, B., Gill, T. (2005). Injuries in women's professional soccer. British journal of sports medicine, 39 (4), 212 - 216. https://doi.org/10.1136/bjsm.2004.011973

Gribble, P. A., Hertel, J., Plisky, P. (2012). Using the star excursion balance test to assess dynamic postural-control deficits and outcomes in lower extremity injury: a literature and systematic review. Journal of athletic training, 47(3), 339-357. https://doi.org/10.4085/1062-605047.3.08

Haksever, B., Düzgün, İ., Deniz, Y., Baltacı, G. (2017). Sağlıklı bireylere standart denge eğitiminin dinamik, statik denge ve fonksiyonellik üzerine etkileri. Gazi Să̆lık Bilimleri Dergisi, 2(3), 40-49. ISSN 2548-0383. https://doi.org/10.4085/1062-6050-47.3.08

Hale, S. A., Hertel, J., Olmsted-Kramer, L. C. (2007). The effect of a 4-week comprehensive rehabilitation program on postural control and lower extremity function in individuals with chronic ankle instability. Journal of orthopaedic \& sports physical therapy, 37(6), 303-311. https://doi.org/10.2519/jospt.2007.2322

Heidt, R. S., Sweeterman, L. M., Carlonas, R. L., Traub, J. A., Tekulve, F. X. (2000). Avoidance of soccer injuries with preseason conditioning. The American journal of sports medicine, 28(5), 659-662. https://doi.org/10.1177/03635465000280050601

Hertel, J., Miller, S. J., \& Denegar, C. R. (2000). Intratester and intertester reliability during the Star Excursion Balance Tests. Journal of sport rehabilitation,9 (2), 104 - 116. https://doi.org/10.1123/jsr.9.2.104

Kabul, E. G., Çalık, B. B., Aslan, U. B., Ünver, F., Büyükturan, Ö., Büyükturan, B., Sönmezer, E. (2018). Sağlıklı gençlerde kısa dönem dinamik stabilizasyon eğitiminin esneklik, kassal endurans ve dinamik denge üzerine etkileri: rastgele kontrollü çalışma. Journal of Exercise Therapy and Rehabilitation, 5(1), 1-8. e-ISSN 2148-8819.

Kiesel, K., Plisky, P. J., Voight, M. L. (2007). Can serious injury in professional football be predicted by a preseason functional movement screen?. North American journal of sports physical therapy: NAJSPT, 2(3), 147. PMID: 21522210.

Kinzey, S. J., Armstrong, C. W. (1998). The reliability of the star-excursion test in assessing dynamic balance. Journal of orthopaedic \& sports physical therapy,27(5), 356-360. https://doi.org/10.2519/jospt.1998.27.5.356

Koçak, U. Z., ve Ünver, B. (2019). Kadın futbolcularda yaralanma riski belirleyicileri olarak fonksiyonel hareket analizi ve y denge testi arasındaki ilişkinin incelenmesi. Spor Hekimliği Dergisi, 54(1), 001-008. https://doi.org/10.33468/sbsebd.108

Ljungqvist, A., Jenoure, P., Engebretsen, L., Alonso, J. M., Bahr, R., Clough, A., Meeuwisse, W. (2009). The international olympic committee (10c) consensus statement on periodic health evaluation of elite athletes march 2009. British journal of sports medicine, 43(9), 631-643. https://doi.org/10.1136/bjsm.2009.064394 
Olmsted, L. C., Carcia, C. R., Hertel, J., Shultz, S. J. (2002). Efficacy of the star excursion balance tests in detecting reach deficits in subjects with chronic ankle instability. Journal of athletic training, 37(4), 501. PMID: 12937574.

Plisky, P. J., Gorman, P. P., Butler, R. J., Kiesel, K. B., Underwood, F. B., Elkins, B. (2009). The reliability of an instrumented device for measuring components of the star excursion balance test. North American journal of sports physical therapy: NAJSPT, 4(2), 92. PMID: 21509114.

Plisky, P. J., Rauh, M. J., Kaminski, T. W., Underwood, F. B. (2006). Star Excursion Balance Test as a predictor of lower extremity injury in high school basketball players. Journal of Orthopaedic \& Sports Physical Therapy, 36(12), 911-919. https://doi.org/10.2519/jospt.2006.2244

Shaffer, S. W., Teyhen, D. S., Lorenson, C. L., Warren, R. L., Koreerat, C. M., Straseske, C. A., \& Childs, J. D. (2013). Y-balance test: a reliability study involving multiple raters. Military medicine, 178(11), 1264-1270. https://doi.org/10.7205/milmed-d-13-00222 


\section{Ek: Etik Kurul Raporu}

T.C. CUKUROVA ÖNIVERSITESI TIP FAKÜLTESI GIRISSIMSEL OLMAYAN KLINIK ARASTIRMALAR ETIK KURULU

\begin{tabular}{|c|c|c|}
\hline Toplantı Sayısı & Tarih \\
\hline 95 & & 10 Ocak 2020 \\
\hline
\end{tabular}

KARAR NO 57- Cukurova Úniversitesi Beden Egitimi ve Spor Yaksekokulu'nda, Doktor Ögretim Ôyesi Cenab Tưrkeri, Yüksek Lisans Ögrencisi Bilgihan Büyüktaş, Yüksek Lisans Öğrencisi Banşcan Öztürk tarafindan yürütülmesi öngörülen, "Y Dinamik Denge Testi Güvenirlik Calıșması" başlıklı proje araştırma etiği yönünden değerlendirildi. Toplantıya katılan ũyelerin oybirliḡiyle uygun olduğuna karar verildi.

\begin{tabular}{|c|c|c|}
\hline BASSKAN & $\begin{array}{l}\text { Prof Dr Selim Kadıoğlu } \\
\text { Tıp Tarihi ve Etik Anabilim Dalı }\end{array}$ & \\
\hline \multirow{8}{*}{ ÜYELER } & $\begin{array}{l}\text { Prof Dr Davut Alptekin } \\
\text { Tıbbi Biyoloji Anabilim Dalı }\end{array}$ & \\
\hline & \begin{tabular}{|l|} 
Prof Dr Dinçer Yıldızdaş \\
Cocuk Sağglığı ve Hastalıkları Anabilim Dalı \\
\end{tabular} & \\
\hline & \begin{tabular}{|l|} 
Prof Dr Gülşah Seydaoğlu \\
Biyoistatistik Anabilim Dalı \\
\end{tabular} & Toplantıya Katılmadı \\
\hline & \begin{tabular}{|l|} 
Prof Dr Gürhan Sakman \\
Genel Cerrahi Anabilim Dalı \\
\end{tabular} & \\
\hline & $\begin{array}{l}\text { Prof Dr Murat Gündüz } \\
\text { Anesteziyoloji ve Reanimasyon Anabilim Dalı }\end{array}$ & \\
\hline & $\begin{array}{l}\text { Doç Dr Ezgi Özyılmaz } \\
\text { Gögüs Hastalıkları Anabilim Dalı }\end{array}$ & \\
\hline & $\begin{array}{l}\text { Av. Zehra Bulut } \\
\text { Hukukçu Ûye }\end{array}$ & Toplantıya Katılmadı \\
\hline & $\begin{array}{l}\text { Dr Neşe Kayrın } \\
\text { Kurum Dışı Ûye }\end{array}$ & Toplantıya Katılmadı \\
\hline
\end{tabular}

Çukurova Universitesi Tip Fakültesi Dekanlık Binası, Balcalı 01330 Adana Telefon: 03223386060 dahili 3465, Faks: 03223386722 